\title{
The Effectiveness NGN/IMS Networks in the Establishment of a Multimedia Session
}

\author{
Ibrahimov G. Bayram ${ }^{1}$, Ismaylova R. Sevinc ${ }^{2}$ \\ ${ }^{1}$ Department of Multichannel Telecommunication Systems, Azerbaijan Technical University, Baku, Azerbaijan \\ ${ }^{2}$ Institute of Control Systems, National Academy of Sciences of Azerbaijan, Baku, Azerbaijan
}

Email address:

i.bayram@mail.ru (B. G. Ibrahimov), sevinc_ism@hotmail.com (S. R. Ismaylova)

\section{To cite this article:}

Ibrahimov G. Bayram, Ismaylova R. Sevinc. The Effectiveness NGN/IMS Networks in the Establishment of a Multimedia Session. American Journal of Networks and Communications. Vol. 7, No. 1, 2018, pp. 1-5. doi: 10.11648/j.ajnc.20180701.11

Received: December 1, 2017; Accepted: December 12, 2017; Published: January 31, 2018

\begin{abstract}
The subject of the study is multiservice communication network using the concept NGN (Next Generation Network) based on the open network architecture IMS (Internet Protocol Multimedia Subsystem), supporting a wide range of services. The basis of this architecture is the IMS core, consisting of a set of specialized modules responsible for various functions for customer service. The purpose of the article is to analyze the existing technical capabilities of the IMS multimedia messaging subsystem and perspective solutions for the functioning of the NGN/IMS network efficiency in providing multimedia services. The effectiveness NGN/IMS networks during the establishment of a multimedia session was analyzed and the functional architecture of the IMS multimedia messaging subsystem that determine the interaction of NGN signaling systems an protocols was explored. One of the important requirements for the IMS subsystem is the maintenance QoS (Quality of Service). A mathematical model for estimating the quality of communication services using a system $G I / G / 1 / N_{б н}$ based on the theory of diffusion approximation is proposed. On the basis of the model analytical expressions are obtained, which allow evaluating the performance indicators of the Triple Play service.
\end{abstract}

Keywords: NGN/IMS Networks, Multimedia Session, SIP Protocol, IMS Subsystem, Signaling Traffic

\section{Introduction}

Intensive development of the multiservice communication networks packet switched using the architectural concept NGN requires improving probable time response characteristics of a subsystem of a multimedia communication IMS (Internet Protocol Multimedia Subsystem) in case of establishment connections and when rendering the multimedia services providing QoS packets of a traffic systems and signaling protocols [1-3].

The system and technical analysis showed $[4,5]$ that a great number telecom operators, the providing multimedia services, the management systems transmission of the official traffic realizing Triple play services such as voice services with a possibility activation multimedia applications, video telephony, IPTV, voice and high speed Internet access use. Such approach allows to integrate different services, gives ample opportunities personalization and increase in number multimedia services.
For provision telecommunication companies abovementioned services by operators in multiservice communication networks it is necessary to consider quality of service QoS of the official and useful traffic and perception (Quality of Experience, QoE) which is estimated by several criteria of efficiency [6]. Support of quality of service QoS \& QoE is the key requirement to a subsystem IMS and an important index efficiency of the NGN/IMS networks in case of establishment of a multimedia session. However, the matter is studied insufficiently well and remains low-probed [1-3, 7-12].

\section{Problem Definition of a Research}

It is known [1-3] that time connection establishment is the major QoS an index of the NGN/IMS networks and is defined since the moment when the terminal of the calling party was transferred by all message necessary for connection establishment, till the moment when this terminal equipment received a signal of a status of the terminal of the called party. 
On the basis of a research it is defined $[4,10]$ that the considered NGN/IMS network in case of service flows packets of a traffic systems and signaling protocols represents the single-channel queuing system (QS) of type $G I / G / 1 / N_{\sigma н}$ with limited queue (on Kendall- GI-s designations arbitrary distribution with independent intervals between arrivals requests).

Considering the nature of a network traffic of systems and the signaling protocols NGN/IMS, the mathematical model (MM) of multimedia service considering joint service of the official and useful traffic when rendering multimedia services is offered.

Mathematical statement of the task offered MM for assessment indices efficiency of the NGN/IMS networks in case establishment of a multimedia session is described by the following target function:

$$
\left.E_{\text {eff }}\left(\lambda_{i}, \eta_{i}\right)=\left\{\underset{i}{\arg \min }\left(T_{i . u s t}(\lambda)\right)\right], \quad i=\overline{1, n}\right\},
$$

in case of the following restrictions

$$
T_{i . d} \leq T_{i . d . a l l .}, T_{i . f a i .} \leq T_{i . f a i . a l l .}, T_{i . \exp } \leq T_{i . \exp . \text { all. }}, i=\overline{1, n}
$$

where $T_{i . d}$ - the average time of a time delay by transmission $i-$ th a flow of packets; $T_{i \text {.exp }}-$ the average time of waiting in queue in case of service $\mathrm{i}$ - th a flow of packets; $\eta_{i}-$ coefficient effective use of network resources NGN/IMS by transmission $i$ - th a flow of packets; $T_{i \text {.fai. }}$ - average values of the time interval of failures between packets of the stream of i-th traffics; $T_{i . \text {.all. }}, T_{i . \text { faiall. }}$ and $T_{i \text {.exp.all. }}-$ respectively, admissible values temporal indices of the NGN/IMS networks by transmission $\mathrm{i}$ - th a flow packets, $i=\overline{1, n}$.

Expressions (1) and (2) define an entity of the considered new approach, based on a mathematical model assessment quality of communication services considering indices efficiency of the NGN/IMS networks and feature methods of diffusion approximation.

Therefore, offered MM, describes quality of functioning of the NGN/IMS networks in case of establishment of a multimedia session which represents QS of the general type $G I / G / 1 / N_{б н}$ with limited queue and with possible multirate servicing of packets of a signal traffic of the SIP $\lambda_{\text {sip }}$, Sigtran $\lambda_{\text {sig }}$ and Diameter $\lambda_{\text {dia }}$ protocol in case of critical loading $\rho \leq 1$.

In this operation the model of functioning of efficiency of the NGN/IMS networks when rendering multimedia services is analyzed, such as voice services with a possibility of activation of multimedia applications.

\section{The Diagram Functioning of the Researched Model of the Multiservice NGN/IMS Networks}

Based on the analysis of quality operation of the multiservice NGN/IMS c networks use of systems and protocols SIP terminals defined the functional architecture IMS which contains the following levels $[1,7]$ :

1. Access level and transport;

2. Level control of sessions;

3. Service layer and applications.

For the description of a subsystem of a multimedia communication of IMS using the server of house subscribers of HSS (HSS - Home Subscriber Server) in figure 1 the diagram functioning model of service of a traffic multimedia services on the NGN/IMS network is offered.

The system and technical analysis shows that the procedure establishment of a multimedia session is initiated by the multi-function terminal printer and the gateway network access, transmitting a request of INVITE switching nodes with use protocols IP/MPLS, through access networks to services and gateways.

Follows from the diagram that an algorithm reception and service of a traffic multimedia communications IMS begins on the NGN/IMS network at the level of an access network as a multiservice node access.

Further traffic multimedia service are serviced by means of the signal gateway, an IMS network kernel with use of the server house subscribers of HSS and transferred to the AS (Application Server) which interacts with a logic function of S-CSGF, I-CSGF and P-CSGF (Serving, Interrogating, Policy - Call Session Control Function) according to the SIP protocol. Here, the SIP protocol is used for establishment, control and uncoupling communication sessions. For procedures of authorization, authentication and account in IMS it is used, also the Diameter protocol.

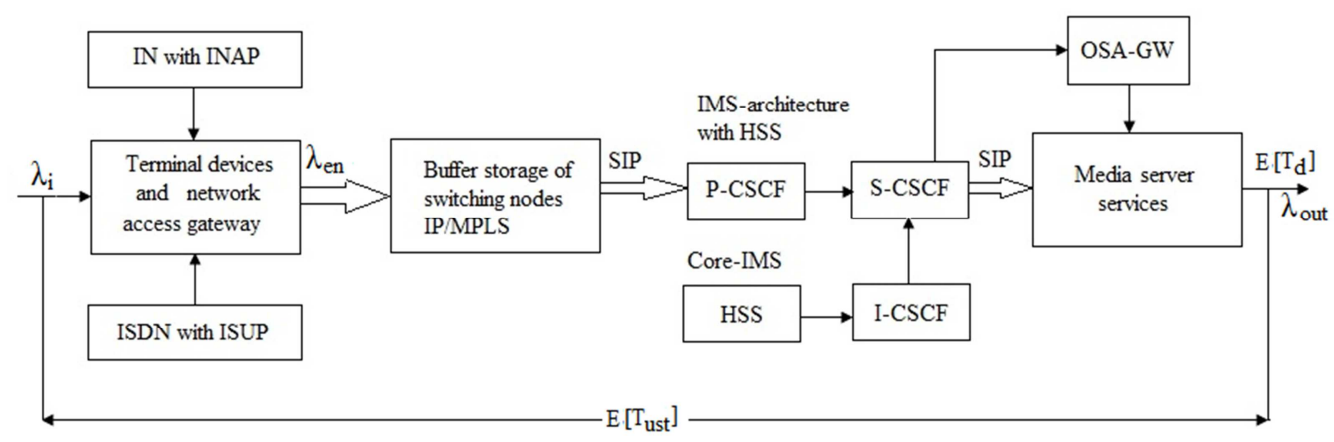

Figure 1. The skeleton diagram functioning model of service of a traffic on the NGN/IMS network. 
From the description of the diagram functioning model of service of a traffic on the NGN/IMS network in case establishment of a multimedia session it is visible that the characteristic of a flow of packets of a traffic signaling protocols is described by the following functional dependence:

$$
\lambda=W\left(\lambda_{\text {en. }}, \lambda_{\text {ser. }}, \lambda_{\text {ref. } .}, \lambda_{\text {out. }}\right)
$$

where $\lambda_{\text {en. }}, \lambda_{\text {ser. }}, \lambda_{\text {ref. }}, \lambda_{\text {out. }}-$ respectively, the speed of the entering, serviced, refused and outting flow packets traffic protocol of the NGN/IMS networks in case establishment multimedia sessions.

IMS kernel with use HSS realizes functions of a request, function of a proxy server and function session management communication. After reception and processing, requests and responses of service by a multimedia subsystem IMS arrive on an application server of services and the media server of service.

\section{Assessment Indices Efficiency of the NGN/IMS Networks}

From brought above - the described principle action of the NGN/IMS c networks use of SIP terminals follows that operation when rendering multimedia services and in case establishment of a session can be considered as single-phase unilinear QS with a finite volume of the buffer storage $N_{b s}$.

We assume that on an input of the buffer storage (BS) switching nodes of the NGN/IMS network the flow packets of a traffic signaling protocols with certain characteristics arrives. Such model can be analyzed as QS of the general type $G I / G / 1 / N_{b s}$ with limited queue.

On the basis model, for the purpose assessment time response characteristics of the NGN/IMS networks in case establishment of a multimedia sessions the approximate analytical method diffusion approximation which accuracy lies in acceptable limits can be used [10].

The idea of a method diffusion approximation, consists that distribution $P_{k}$ queue length in system $G I / G / 1 / N_{б н}$ with limited queue in case of the general loading $\rho$, is approximated by distribution of the following look:

$$
P_{k}=\left\{\begin{array}{lll}
0, & \text { for } k=0 \\
\left(1-\rho^{*}\right)\left(\rho^{*}\right)^{k-1}, & \text { for } k \geq 0
\end{array}\right.
$$

here $P_{k}$ - characterizes that in each time point the system has distributions probabilities phase statuses; $\rho^{*}$ - duty ratio switching nodes of the NGN/IMS networks.

Considering square coefficients variations distribution $C_{A}^{2}$ intervals between the arriving multimedia messages and distribution lengths multimedia messages $C_{B}^{2}$ the duty ratio expresses as follows:

$$
\rho^{*}=\exp \left[\left(\mu \cdot C_{S}^{2}-\lambda \cdot C_{A}^{2}\right) / 2(\lambda-\mu)\right],
$$

where $\mu$ - intensity service of a traffic.

Considering the above, in this system, it is supposed that the speed arrival flows of the official traffic $\lambda_{i}, i=\overline{1, n}$ differs from Poisson, and process of service from the exponential distribution law. At the same time loading in this QS is defined by the following expression:

$$
\rho=\sum_{i=1}^{n} \lambda_{i} \cdot\left[D_{i} / C_{S}(i)\right] \leq 1, i=\overline{1, n},
$$

where $D_{i}-$ dispersion working hours $i$-th traffics; $\lambda_{i}-$ speed of the arriving flows official i-th traffics; $C_{B}(i)-$ coefficient of a variation distribution lengths packets official $i$-th traffics, $i=\overline{1, n}$.

On the basis of the theory and methods diffusion approximation $[8,10]$, joint speed of the quitting flow of the official traffic signaling protocols of the NGN/IMS networks is defined by the following expression:

$$
\lambda_{\text {out. }}=\lambda_{\text {en. }}-\lambda_{\text {ser. }}-\lambda_{\text {ref }}
$$

Considering QS of the general type $G I / G / 1 / N_{b s}$ with limited queue and using parameters methods diffusion approximation, it is possible to define characteristics of a day off flows packets of a traffic switching nodes of the NGN/IMS networks and also characteristics failures flows packets of a traffic.

Main objective of a research model $G I / G / 1 / N_{b s}$ with limited queue is minimization of the time response characteristics access to multimedia services provided by the NGN/IMS network in case establishment of a multimedia session.

We will assume that because failures in system $\lambda_{\text {ser. }}=0$. At the same time using the equations balance (7) it is possible to define mean value $E\left[T_{\text {fai }}\right]$ - time interval between packets traffic in a failure flow which expresses as follows:

$$
E\left[T_{\text {fai. }}\right]=\frac{\lambda_{\text {en. }} \cdot \lambda_{\text {out. }}}{\lambda_{\text {en }}-\lambda_{\text {out. }}} \leq T_{\text {fai.all } .}
$$

Thus, on the basis model $G I / G / 1 / N_{b s}$ a received expression (8) characterizes the average time failures in case service of a traffic and is QoS \& QoE index $[5,12]$.

\section{Determination Average Time Establishment of a Multimedia Session}

Taking into account feature QS of the general type $G I / G / 1 / N_{b s}$ with limited queue, the average length queue in $E\left[L_{c p}\right] \mathrm{BS}$ switching nodes of the NGN/IMS networks can be determined by a formula Litlla by the following 
expression [6]:

$$
E\left[L_{c p}\right]=\lambda_{e n .} \cdot\left\{1-E\left[P_{f a i .}\right]\right\} \cdot E\left[T_{\text {exp. }}\right],
$$

It is necessary to mark that expressions (5) and (6) define probability time characteristics systems and signaling protocols of the NGN/IMS networks and are figures merit of service QoS of a multiservice traffic.

On the basis of the offered MM, average time establishment of a multimedia session for rendering of services Triple Play (a triple packet) corresponding to the average time stay packets of a traffic in switching node of the NGN/IMS network expresses as follows:

$$
E\left[T_{\text {ust. }}\right]=\left\{E\left[T_{\text {exp. }}\right]+\mu^{-1}\right\} \cdot\left(1-P_{\text {fai. }}\right)
$$

Expression (10) is an index efficiency of the NGN/IMS network in case establishment of a multimedia sessions and respectively, is one key indicators functioning of a signaling network.

\section{Results of the Numerical Analysis}

Results of the numerical analysis on a figure 2 the dependence average time establishment of a multimedia sessions is provided to the NGN/IMS networks from intensity of the entering flow packets of a signal traffic and transmission rate of a multimedia traffic.

The analysis family graphic dependence $E\left[T_{u s t .}\right]=W\left(\rho, \lambda_{e n}, V_{c k}\right)$ shows that with increase in intensity of the entering flow packets of a traffic of services Triple Play on the NGN/IMS network with use home subscribers servers HSS, the average time establishment of a multimedia sessions in case of the set speed of the $V_{c k} \geq(2, \ldots, 100) \mathrm{Mbit} / \mathrm{s}$, $P_{\text {fai. }} \leq 1,5 \cdot 10^{-6}$ and duty ratio increase $\rho=(0,50, \ldots, 0,65)$.

Besides, the provided diagram shows that the average time connection establishment by provision of service Triple Play does not exceed 3,6 s and also begins to increase sharply, since values of intensity near $(16, \ldots, 24) \mathrm{pac} / \mathrm{s}$ that is connected to violation of conditions (6) of existence of the stationary mode.

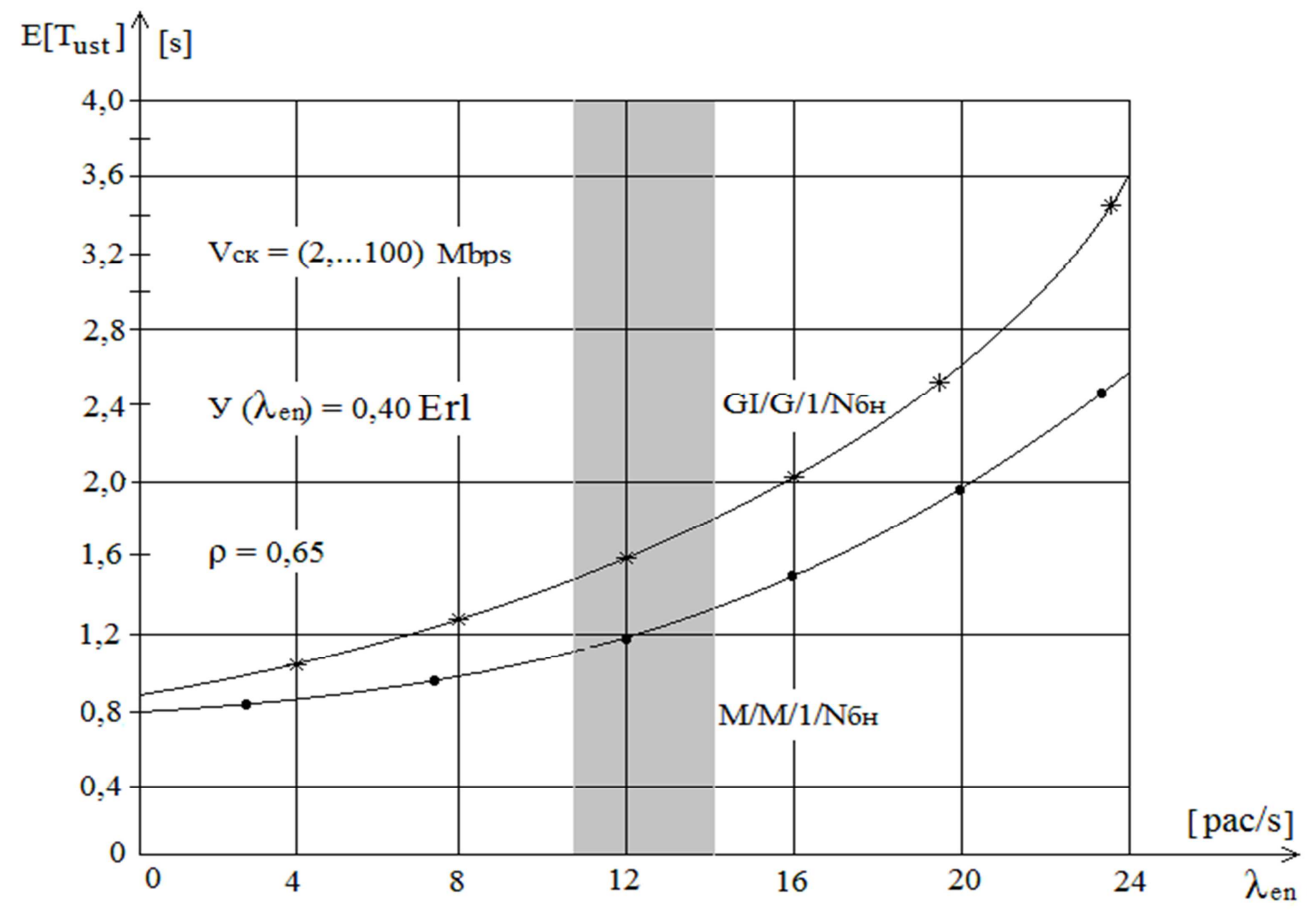

Figure 2. Graphic dependence average time establishment multimedia sessions on the NGN/IMS network from intensity of the entering flow packets of a signal traffic.

We will note that in the specified conditions time connection establishment will not exceed $E\left[T_{\text {ust. }}\right] \leq 4 \mathrm{~s}$, in case of the maximum intensity loading $Y\left(\lambda_{e n}\right) \leq 0,4$ Erlanq in an hour to the greatest loading.

Further, from the graphic in figure 2 it is seen that in the real range of loads $Y\left(\lambda_{e n}\right) \leq(0,15, \ldots, 0,45) E r l$. (in the chart is marked - the area normal load, where $\lambda_{\text {en }}=10, \ldots, 14$ pac. $/ \mathrm{s}$ ), the average connection establishment time is in the range $1,2, \ldots, 1,5 \mathrm{~s}$.

Thus, a comparative analysis of the QS of the general type and $G I / G / 1 / N_{b s}$ and $M / M / 1 / N_{b s}$ shows that the contribution of the IMS core to delays in setting up a multimedia session is significant and should be taken into account when designing NGN / IMS networks.

\section{Conclusions}

The analysis of the functional architecture of NGN in its implementation based on IMS technology for the study multimedia sessions was carried out and a new approach based on the mathematical model of the quality assessment communication services was proposed.

As a result of a research it is offered MM in the form QS 
of the general type $G I / G / 1 / N_{b s}$ with queues which is based on the approximate method diffusion approximation.

The analytical expressions allowing to evaluate figures merit of service QoS \& QoE of a multiservice traffic and to analyze probability time characteristics of the NGN/IMS networks in case establishment of a multimedia session are received.

\section{References}

[1] Bosse J. G., Devetak F. U. Signaling in Telecommunications Networks. 2nd Ed. New York: Wiley, 2007.-830 P.

[2] Mika P., Georg M., Hisham K., Aki N. IMS. IP Multimedia Concepts and Services in the Mobile Domain. John Wiley \& Sons Ltd., 2004. -419 P.

[3] Hannan M., Mustapha A., Hussain A., Basri H. Intelligent Bus Monitoring and Management System// Proceedings of the World Congress on Engineering and Computer Science (WCECS), San Francisco, USA, 2012.

[4] Ibrahimov B. G., Ismaylova S. R. Research efficiency of integrated communication networks with use of signaling system // The IV- International Conference «Problems of Cybernetics and Informatics». Vol. 1. PCI-12. Baku, Azerbaijan. 2012. - P. 71-74.

[5] 3GPP, "Technical Specification Group Services and System
Aspects; Network Architecture (Release 5)," 3GPP, Tech. Rep. TS23. 002, March 2002.

[6] Ibrahimov B. G., Mamedov Sh. M. The Study and Estimation of the Performance Attributes of Terminal Hardware for a Link in a Multiservice Communication Network //Automatic Control and Computer Sciences. USA. 2010. Vol. 48. No. 6.P. 54-59.

[7] Rosenberg J., Schulzrinne H., Camarillo G., Peterson J., Sparks R., Handley M., and Schooler E., "SIP: Session Initiation Protocol,” RFC 3261 IETF, June 2002.

[8] Kleinrock L. Queuing Systems. Vol. I. Theory, Wiley, New York, 1975.

[9] A. Kist and R. J. Harris, "SIP signalling delay in 3GPP." Sixth International Symposium on Communications Interworking, 2002. -P. 211-222.

[10] Ward, A. R. A diffusion approximation for a GI/G/1 queue with balking or reneging / A. R. Ward, P. W. Glinn // Queueing Systems. - 2005. - V. 50, № 4. - P. 371-400.

[11] Zhengxi Wei. Route Research for Ad Hoc Network. American Journal of Networks and Communications. Vol. 3, No. 6, 2014, pp. 73-76.

[12] Mammadov H. A., Ibrahimov B. G. Efficiency of methods forecasting of the office traffic signaling systems with use technologies of neural networks // The 4-rd World Conference on Soft Computing (WConSC-14). Berkeley, California, USA. 2014. -P. 141-145. 